\title{
What can we do to Prevent Infections in Adult Patients with Rheumatic Diseases?
}

\section{Patricia Richi ${ }^{*}$ and Santiago Munoz-Fernandez}

Rheumatology Service Hospital, Universitario Infanta Sofía, Universidad Europea de Madrid, Spain

*Corresponding author: Patricia Richi, Rheumatology Service Hospital, Universitario Infanta Sofía, Universidad Europea de Madrid, Spain, Tel: 3491740727; E-mail: patricia.richi@salud.madrid.org

Received date: May 31, 2016; Accepted date: July 05, 2016; Published date: July 11, 2016

Copyright: (c) 2016 Richi P, et al. This is an open-access article distributed under the terms of the Creative Commons Attribution License, which permits unrestricted use, distribution, and reproduction in any medium, provided the original author and source are credited.

\section{Introduction}

The development of biological therapies has changed the evolution and prognosis of Autoimmune Inflammatory Rheumatic Diseases (AIIRD). These treatments allow better outcomes and clinical remission to be achieved in a significant proportion of subjects. However, immunomodulatory drugs, as well as the underlying AIIRD, are associated with an increased risk of infections, both opportunistic and non-opportunistic [1].

Modifications in the immune regulation process have been described in rheumatic disease such as Rheumatoid Arthritis (RA) and Systemic Lupus Erythematosus (SLE). These modifications may contribute not only to the autoimmune response but to the immunodeficiency observed in these patients. In RA subjects it has been communicated an altered CD4 $\mathrm{T}$ cell homeostasis, with $\mathrm{T}$ cell repertoire aberrations and the emergence of oligoclonal populations with lost in $\mathrm{T}$ cell diversity that will lead to defective immune responses to selected antigens [2]. In Systemic Lupus Erythematosus (SLE), other changes that may predispose to infection, such as breakdown of mechanisms that maintain T- and B-cell tolerance, failure to remove autoreactive $\mathrm{B}$ cells, decreased phagocytosis, reduced complement, cytopenia and a deficiency in serum protein mannosebinding lectin (a key molecule in innate immunity), have been reported [3].

\section{Immunosuppressive Treatments and Infections}

Disease-modifying anti-rheumatic drugs (DMARDs) like leflunomide, azathyoprine, cyclophosphamide and specially methotrexate, are used promptly in the treatment of AIIRD. On the other hand, biological therapies, including Tumor Necrosis Factor blockers, Rituximab, Abatacept, Tocilizumab, and the newer agent that are becoming available, are being used earlier in the course of AIIRD. These immunosuppressive drugs, as well as corticosteroids, increase the risk of infection, in such a way that infections are one of the main co-morbidities in patients on immunosuppressive therapies. According to Gil Prieto et al. a total of 10,861 hospital discharges following pneumococcal disease in adults were reported in Spain during 2011, with an annual hospitalisation incidence of 0.285 (95\% confidence interval (CI): $0.280-0.291$ ) per 1,000 population over 18 years. The case-fatality rate was $8 \%$ and the estimated cost of these hospitalisations in 2011 was over 57 million euro. Among other factors, immunosuppression increased the risk of hospitalisation in patients with pneumococcal pneumonia as well as case-fatality [4].

Bernatsky et al. analysed the risk of infection related to drug exposure in a large cohort of RA patients. In a case-control design nested within a cohort of 23,733 RA patients matching on age and gender and adjusted for comorbidity and physician use, the authors estimated the effect of specific drugs on the Rate Ratio (RR) for infections requiring hospitalisation. They found the highest risk with current exposures to cyclophosphamide (RR: 3.26, 95\% CI: 2.28-4.67) and systemic glucocorticoid agents (RR: $2.56,95 \%$ CI: 2.29-2.85), a moderate risk increase in the case of azathioprine (RR: 1.52, 95\% CI: 1.18-1.97) and some evidence of an increased risk of pneumonia due to methotrexate (RR: $1.16,95 \% \mathrm{CI}: 1.02-1.33$ ). The authors did not report information about glucocorticoids doses [5]. In SLE patients, the use of steroids and cyclophosphamide, both with a dose-dependent effect, has been described as the strongest extrinsic risk factor for infections [6]. A nested case-control study described how prednisone even at moderate doses increases the risk of infections, whilst antimalarial have a protective effect. In this study, the median prednisone dose in patients with major infections was $7.5 \mathrm{mg} /$ day [7].

Patients with antineutrophil cytoplasmic antibody (ANCA) associated vasculitis present a high risk of severe infections, sometimes fatal [8]. Prolonged corticosteroid treatment has been identified as one of the underlying factors [9]. Biological therapies increase the possibility of serious infections, with varying effects on the safety profile depending on their mechanism of action. Abatacept, an inhibitor of co-stimulatin of $\mathrm{T}$ cells, and rituximab, an inductor of incomplete B cell depletion, have shown a similar risk of hospitalised bacterial infections as the Tumor Necrosis Factor blockers, etanercept or adalimumab and lower than infliximab [10].

Cobo-Ibanez et al. analyzed RA or other immune-mediated connective tissue diseases (ICTD) patients on anti-TNF or rituximab included in the Spanish registry BIOBADASER 2.0 (2000-2011), in order to identify predictors of serious infections (SI) [11]. They found age, co morbidities, corticosteroids and ICTD different to RA were all independently associated with SI (Table 1).

One study analysed the infection risk in RA and Spondyloarthropathy patients taking DMARDs, corticosteroids or TNF- $\alpha$ antagonists. Female gender and disease activity were associated with a higher risk of infection. The combination of anti-TNF- $\alpha$ and corticosteroids was found to be the most "pro-infective" treatment, even higher than this combination plus DMARDs. Corticosteroids reduce TNF- $\alpha$ production, while anti-TNF- $\alpha$ inactivates it. The synergic effect of both drugs enhances the anti-inflammatory response but increases the risk of infection.

On the other hand, reduced proliferation of bacteria and also viruses, have been described in association to decreased activity of the enzyme methylenetetrahydrofolate reductase. This reduced activity has been communicated in relation with a common polymorphism in this enzyme gene. MTX presents an anti-methylenetetrahydrofolate 
reductase effect, which may explain, in part, its safer profile in terms of infection [12].

\begin{tabular}{|l|l|}
\hline Risk factor & IRR $[95 \%$ Cl] \\
\hline Chronic obstructive pulmonary disease & $3,53(2,25-5,53)$ \\
\hline Interstitial lung disease & $2,88(1,93-4,3)$ \\
\hline ICTD VS. RA & $1,96(1,06-3,65)$ \\
\hline Renal failure & $1,87(1,02-3,41)$ \\
\hline Corticosteroids & $1,74(1,32-2,28)$ \\
\hline High Blood Pressure & $1,65(1,22-2,23)$ \\
\hline Age & $1,02(1,01-1,03)$ \\
\hline
\end{tabular}

Table 1: Factors related to Severe infections (SI) in patients with Rheumatoid Arthritis (RA) or an Immune-mediated Connective Tissue Diseases (ICTD) other than RA, (multivariate analyses).

Besides the treatments used the underlying diseases constitute another risk factor for serious infections. Many studies have focused on this line of research. Doran et al. compared the frequency of infections in RA patients and individuals without RA. After adjusting for confounder factors, such as age, sex, smoking status, leukopenia, corticosteroid use and diabetes mellitus, the study showed that RA patients were at twice the risk of developing an objectively confirmed or serious infection requiring hospitalisation. Sites of infection with the highest risk ratios were bone, joints, skin, soft tissue and the respiratory tract [13].

Rheumatoid Arthritis has been identified as an independent risk factor for community acquired pneumonia [14]. Blumentals et al. found an increased incidence of seasonal influenza and its complications (pneumonia, stroke, myocardial infarction or neurological disorder on or up to 30 days after the influenza diagnosis) in patients with RA compared with matched controls [15].

In a multicentre Spanish registry (RELESSER) including over 4,000 patients with SLE or incomplete SLE (iSLE, patients that fulfilled 3 of the 1997 ACR SLE criteria), 20.8\% of SLE patients and 9.6\% of iSLE patients suffered severe infections [16]. Using linked hospital data from two data sets (1963 to 2008, 1999 to 2008); Wotton et al tried to determine if individuals with a range of immune-mediated diseases had an increased risk of developing pneumococcal disease [17]. They found a significant increase in the incidence of pneumococcal disease among individuals with AIIRD such as RA, Sjogren's syndrome, Ankylosing Spondylitis (SpA), dermatomyositis/polymyositis, and also in Crohn's disease and ulcerative colitis. A particularly high risk was found for patients with polyarteritis nodosa, scleroderma and SLE.

\section{Vaccines}

Vaccination is one of the most effective measures to prevent infections, prompting the European League Against Rheumatism (EULAR) to draw up some recommendations for all AIIRD patients, not only for those who are on biological therapies (Table 2) $[18,19]$. The EULAR calls on physicians to assess the vaccination status in the initial work-up to ensure that the vaccination recommendations for the general population are covered, and then to administer those proposed for immunosuppressed subjects. It is also important to find out if any adverse events or AIIRD flare up occurred following previous vaccinations, since these could constitute (relative) contraindications in the case of future vaccinations.

\begin{tabular}{|c|c|c|}
\hline Vaccination & Immunization schedules & Comments \\
\hline Tetanus and Diphtheria & $\begin{array}{l}3 \text { doses; } 0,1-12 \text { months. } \\
\text { Booster every } 10 \text { years }\end{array}$ & $\begin{array}{l}\text { In accordance with recommendations for the general } \\
\text { population }\end{array}$ \\
\hline Inactivated Influenza & Every year & Strongly considered \\
\hline Pneumococcal" & $\begin{array}{l}\text { 23-valent polysaccharide; } 1 \text { st dose and a booster } 5 \text { years } \\
\text { later. } 13 \text {-valent conjugated } ; 1 \text { dose }\end{array}$ & Strongly considered \\
\hline Human papillomavirus (HPV) & 3 shots; $0-1$ or $2-6$ months after the 1 st & Women under 25 , especially with SLE diagnosis \\
\hline Meningococcal C & Every $3 / 5$ years & In hyposplenic/asplenic patients \\
\hline B Hepatitis & 3 shots: 0,1 and 6 months after the $1^{\text {st }}$ & Consider in patients at risk of contracting the infection \\
\hline
\end{tabular}

Table 2: Vaccination Recommendations for AIIRD patients $[17,18,34]{ }^{*}$ Recommended intervals for sequential use of PCV13 and PPSV23: Pneumococcal vaccine naive: 13-valent and 23-valent 8 weeks later;Person who previously received 23-valent vaccine: 13-valent vaccine at least 1 year later.

Most vaccines confer protection by eliciting Bcell responses and the production of antibodies that are specific for toxic agents, but neutralising immune effectors' responses can also be Tcell mediated. An adequately functioning immune system is required for protective immunity after vaccination. Therefore, the effectiveness of vaccines in these patients is not well known and it seems to depend on the different types of treatment used. This increases the importance of offering an adequate vaccination programme to AIIRD patients as soon as the diagnosis is made and before starting immunosuppressant therapy and physicians need to be aware of this. Actually, vaccination rates in these populations are low in most series. In the study of Pradeep et al. among 105 patients with RA and/or patients taking DMARDs, 37\% had received both the influenza and pneumococcal vaccine, $23 \%$ received the influenza vaccine only, $2 \%$ received the pneumococcal vaccine only, and $38 \%$ neither [20].

In a large retrospective study, that recorded data from 2000 to 2007 , including 46,030 patients with RA and a matching number of controls, 
influenza vaccination was more common in the controls than in those with RA $(10.14 \%$ Vs $7.60 \%, \mathrm{p}<0.0001)$. The RA group had a significantly higher prevalence of pneumopathy $(5.37 \%$ Vs $3.48 \%$, $\mathrm{p}<$ 0.0001 ), with chronic lung disease being another reason to recommend the vaccine [21].

In contrast Dirven reports influenza vaccinations rates in RA subjects of $74 \%$ [22]. Lanternier et al. reported a low influenzavaccination rate $(28 \%)$ among patients with systemic inflammatory disease. In this report, age $>65$ years was significantly associated with influenza vaccination (46\%), but lower than that in the general population of the same age (70\%) [23]. Forty-nine per cent of patients who remembered having received a letter from the National Health Insurance Service encouraging them to have the vaccine were vaccinated compared with $18 \%$ who did not remember $(p<0.001)$. The main reason for non-vaccination was the absence of doctors recommendation (58\%). Public health campaigns directed to doctors and patients should be undertaken to improve vaccinations rates. Informed patients, working together with family doctors, specialists and trained nurses, show better health outcomes, as it has been demonstrated by a special program designed for patients with cardiovascular disease [24]. Interaction between the different health providers and knowledgeable patients would probably increase the adherence to vaccination recommendations between subjects with chronic AIIRD.

Our group found an influenza-vaccination rate of $70 \%$ in a group of 189 patients with AIIRD on biological therapies. With respect to the pneumococcal vaccine, we observed that $58.2 \%$ of patients received the pneumococcal polysaccharide vaccine and only $20.7 \%$ the conjugated vaccine (unpublished data).

\section{Vaccination Effectiveness}

Assessing the effectiveness of vaccines is not easy. From a clinical point of view, the desired endpoint is that those vaccinated present a lower clinical disease rate than non-vaccinated persons, with or without laboratory confirmation.

Kobashigawa et al. studied the relative risk of vaccination for an influenza attack in RA patients. Enrolled subjects completed the selfadministered questionnaires which included their influenza vaccination status and the occurrence of an actual influenza attack during the 2001, 2002, 2003 and 2007 seasons. Combining the four seasonal results, vaccination was associated with a reduced risk of an influenza attack (odds ratio [OR] 0.76, 95\% CI 0.63-0.91, p<0.01). There were no associations between influenza attacks and RA disease activity, treatment with methotrexate (MTX) or corticosteroids [21].

Stojanovich et al. analysed the occurrence of viral respiratory and bacterial infections in influenza-vaccinated SLE and RA patients compared with non-vaccinated patients during the 2003 influenza season. Vaccinated patients had significantly fewer infections. The vaccine was well tolerated in all cases while viral and bacterial infections resulted in the worsening of the main disease [25].

Despite the importance of using clinical endpoints, most studies investigated the efficacy of vaccines by measuring humoral or cellmediated immune responses even though there is a lack of correlation between antibody $(\mathrm{Ab})$ titres and protection in AIIRD patients.

Kostianovsky et al. studied the immunogenicity and safety of seasonal flu vaccine (SFV) and 2009 pandemic A/H1N1 influenza (HFV) vaccine in 199 patients with autoimmune diseases, including different diagnosis (Systemic Necrotising Vasculitides, Behçet's disease, Cryoglobulinaemia, Systemic Sclerosis, SLE, Polymyositis, Dermatomyositis, SpA, RA, Sarcoidosis, Psoriatic Arthritis, Idiopathic Retroperitoneal Fibrosis, Polychondritis, Autoimmune Haemolytic Anaemia and Primary Antiphospholipid Syndrome). Immunogenicity was studied in terms of seroprotection (patients with post-vaccination $\mathrm{Ab}$ titres $>1: 40$ measured by haemagglutination-inhibition assay [HIA]) and seroconversion (a 4-fold HIA-titre increase after immunisation compared to pre-vaccination value). Eighty per cent of patients vaccinated with SFV and $65 \%$ of those who received HFV became seroprotected. No difference was observed according to the diagnoses. The immunosuppressive therapy was not associated with a lower seroprotection level. The number of patients receiving biotherapies was low and this group did show a trend to lower protection rates after both SFV and HFV, compared to immunosuppressed and non-immunosuppressed patients. The authors observed a low number of adverse local and systemic reactions as well as vaccine related flares, concluding that the overall tolerance and safety of SFV and HFV was acceptable [26]. Other authors have also found that corticosteroids or DMARDs, mainly MTX, have no significant influence on influenza vaccination $[27,28]$.

Kapetanovic studied the impact of anti-rheumatic treatment on the immunogenicity of the pandemic H1N1 influenza vaccine in 291 patients with RA or SpA. They found the best response in the SpA group taking anti-TNF monotherapy, where $76 \%$ of patients achieved seroprotection. RA patients on methotrexate, anti-TNF monotherapy or anti-TNF plus MTX as well as SpA subjects on anti-TNF plus MTX showed rates between 45 and $63 \%$ of vaccinees seroprotected. Forty percent of RA patients on abatacept and only $20 \%$ on rituximab achieved post-vaccination HIA titres $>40$ [29].

Other studies also found a modestly impaired antibody response to influenza, with a proportion of post-vaccinated seroprotected patients not significantly diminished by the use of TNF blocking therapies but a clearly worse response in those with rituximab $[27,30]$.

One report analysed antibody increases following pneumococcal vaccination using a 7 -valent conjugate vaccine. A positive antibody response was defined as an antibody response ratio (ARR) (i.e., ratio of post- to pre-vaccination antibody levels) of $\geq 2$. A significantly better ARR was seen among patients in the control group (SpA patients receiving nonsteroidal anti-inflammatory drugs [NSAIDs] and/or analgesics) compared to those in groups treated with MTX or MTX in combination with TNF inhibitors. Among patients treated with TNF inhibitors monotherapy, ARRs for both serotypes were lower numerically, but were not significantly different compared to the controls. Thus, in this study, ongoing MTX treatment was predictive of a reduced response (OR 0.41 [95\% CI 0.24-0.68], $\mathrm{P}=0.001$ ) [31].

The impact of rituximab, abatacept and tocilizumab was also investigated and it was found that abatacept and mainly rituximab were associated with diminished antibody response in RA patients vaccinated with the 7 -valent conjugate vaccine. Thus, pneumococcal vaccination should be encouraged before initiation of rituximab or abatacept treatment [32].

With respect to safety, although there are reports of flare-ups of the underlying disease after vaccination, vaccines appear to be safe for use in patients with AIIRD, particularly in those with a low disease activity.

Salemi and D'Amelio analysed the safety of vaccination in autoimmune diseases. They searched the medical literature from 1947 to 2009 and found data on over 5000 vaccinated patients with 
autoimmune diseases (multiple sclerosis, SLE, RA, insulin-dependent diabetes mellitus, chronic arthritis (CA) in children and vasculitides), mostly referring to the influenza and pneumococcal vaccines. To investigate the safety, only clinical (rate of post-vaccine disease exacerbation as inferred from clinimetric indices) and immunological (rate of post-vaccine autoantibody new onset or rise in titre) autoimmune disease worsening were considered. They found that the inactivated flu vaccine appears to be generally safe for use in RA, children with CA and vasculitides patients. Despite anecdotal cases of disease worsening after vaccination, it also seems generally safe for use in SLE patients provided patients with quiescent disease are chosen. With respect to pneumococcus, the different vaccines analysed (7valent, 14-valent, 23-valent) also appear to be safe in SLE and RA patients [33].

Nevertheless, the numbers of patients with moderate or severe disease included in the safety studies are too small to conclude that vaccination during active disease is safe. Therefore, EULAR guidelines, based on the theoretical risks of disease flare following vaccination in unstable patients with AIIRD, recommend vaccination during stable disease [18].

\section{Conclusions}

AIIRD patients present a high risk of infections, and vaccination is one of the most effective measures to prevent these. Although further studies are required, vaccines appear to be safe and effective in this patient group. Physicians should be familiar with the vaccine schemes and should encourage AIIRD patients to follow the vaccination recommendations.

\section{References}

1. Salmon-Ceron D, Tubach F, Lortholary O, Chosidow O, Bretagne S, et al. (2011) Drug-specific risk of non-tuberculosis opportunistic infections in patients receiving anti-TNF therapy reported to the 3-year prospective French RATIO registry. Ann Rheum Dis 70:616-23.

2. Wagner UG, Koetz K, Weyand CM, Goronzy JJ (1998) Perturbation of the $\mathrm{T}$ cell repertoire in rheumatoid arthritis. Proc Natl Acad Sci U S A. 5: 14447-52.

3. Cuchacovich R, Gedalia A (2009) Pathophysiology and clinical spectrum of infections in systemic lupus erythematosus. Rheum Dis Clin North Am 35:75-93.

4. Gil Prieto R, Pascual-Garcia R, Walter S, Álvaro-Meca A, Gil de Miguel Á, et al.( 2016) Risk of Hospitalization due to pneumococcal disease in adults in Spain. The CORIENNE study. Hum Vaccin Immunother. Feb 22: 1-6.

5. Bernatsky S, Hudson M, Suissa S (2007) Anti-rheumatic drug use and risk of serious infections in rheumatoid arthritis. Rheumatology 46:11571160 .

6. Kang I, Park SH (2003) Infectious complications in SLE after immunosuppressive therapies. Curr Opin Rheumatol 15: 528-534.

7. Ruiz-Irastorza G, Olivares N, Ruiz-Arruza I, Martinez-Berriotxoa A, Egurbide MV, et al. (2009) Predictors of major infections in systemic lupus erythematosus. Arthritis Research \& Therapy 11: R109.

8. Flossmann O (2015) Risks of treatments and long-term outcomes of systemic ANCA-associated vasculitis. Presse Med 44: e251-e257.

9. Goupil R, Brachemi S, Nadeau-Fredette AC, Deziel C, Troyanov Y, et al. (2013) Lymphopenia and treatment-related infectious complications in ANCA-associated vasculitis. Clin J Am Soc Nephrol 8: 416-423.

10. Curtis JR, Yang S, Patkar NM, Chen L, Singh JA, et al. (2014) Risk of hospitalized bacterial infections associated with biologic treatment among US veterans with rheumatoid arthritis. Arthritis Care Res 66: 990-997.
11. Cobo-Ibáñez T, Descalzo MÁ, Loza-Santamaría E, Carmona L, MuñozFernández S, et al. (2014) Serious infections in patients with rheumatoid arthritis and other immune-mediated connective tissue diseases exposed to anti-TNF or rituximab: data from the Spanish registry BIOBADASER 2.0. Rheumatol Int 34: 953-961.

12. Germano V, Cattaruzza MS, Osborn J, Tarantino A, Di Rosa R, Salemi S et al. (2014) Infection risk in rheumatoid arthritis and spondyloarthropathy patients under treatment with DMARDs, corticosteroids and TNF- $\alpha$ antagonists. J Transl Med 12: 77.

13. Doran MF, Crowson CS, Pond GR, O'Fallon WM, Gabriel SE, et al. (2002) Frequency of infection in patients with rheumatoid arthritis compared with controls: a population-based study. Arthritis Rheum 46: 2287-2293.

14. Vinogradova Y, Hippisley-Cox J, Coupland C (2009) Identification of new risk factors for pneumonia: population-based case-control study. Br J Gen Pract 59: e329-338.

15. Blumentals WA, Arreglado A, Napalkov P, Toovey S (2012) Rheumatoid arthritis and the incidence of influenza and influenza-related complications: a retrospective cohort study. BMC Musculoskelet Disord 13:158.

16. Rúa-Figueroa Í, Richi P, López-Longo FJ, Galindo M, Calvo-Alén J, et al. (2015) Comprehensive description of clinical characteristics of a large systemic lupus erythematosus cohort from the Spanish Rheumatology Society Lupus Registry (RELESSER) with emphasis on complete versus incomplete lupus differences. Medicine (Baltimore) 94:267.

17. Wotton CJ, Goldacre MJ (2012) Risk of invasive pneumococcal disease in people admitted to hospital with selected immune-mediated diseases: record linkage cohort analyses. J Epidemiol Community Health 66: 1177-1181.

18. Van Assen S, Agmon-Levin N, Elkayam O, Cervera R, Doran MF, et al. (2011) EULAR recommendations for vaccination in adult patients with autoimmune inflammatory rheumatic diseases. Ann Rheum Dis 70: 414-422.

19. Gómez Reino J, Loza E, Andreu JL, Balsa A, Batlle E, et al. (2011) Consensus statement of the Spanish Society of Rheumatology on risk management of biologic therapy in rheumatic patients. Reumatol Clin 7:284-298.

20. Pradeep J, Watts R, Clunie G (2007) Audit on the uptake of influenza and pneumococcal vaccination in patients with rheumatoid arthritis. Ann Rheum Dis. 66: 837-838.

21. Kobashigawa T, Nakajima A, Taniguchi A, Inoue E, Tanaka E, et al. (2013) Vaccination against seasonal influenza is effective in Japanese patients with rheumatoid arthritis enrolled in a large observational cohort. Scand J Rheumatol 42: 445-450.

22. Dirven L, Huizinga TWJ, Allaart CF (2012) Risk factors for reported influenza and influenza-like symptoms in patients with rheumatoid arthritis. Scand J Rheumatol 41: 359-365.

23. Lanternier F, Henegar C, Mouthon L, Blanche P, Guillevin L, et al. (2008) Low Influenza-vaccination rate among adults receiving immunosuppressive therapy for systemic inflammatory disease. Ann Rheum Dis 67:1047.

24. Ciccone MM, Aquilino A, Cortese F, Scicchitano P, Sassara M, et al. (2010) Feasibility and effectiveness of a disease and care management model in the primary health care system for patients with heart failure and diabetes (Project Leonardo) Vasc Health Risk Manag 6:297-305.

25. Stojanovich L (2006) Influenza vaccination of patients with systemic lupus erythematosus (SLE) and rheumatoid arthritis (RA). Clin Dev Immunol 13: 373-375.

26. Kostianovsky A, Charles P, Alves J-F, Goulet M, Pagnoux C, et al. (2012) Immunogenicity and safety of seasonal and 2009 pandemic A/H1N1 influenza vaccines for patients with autoimmune diseases: a prospective, monocentre trial on 199 patients. Clin Exp Rheumatol S83-S89.

27. Kubota T, Nii T, Nanki T, Kohsaka H, Harigai M, et al. (2007) Anti-tumor necrosis factor therapy does not diminish the immune response to influenza vaccine in Japanese patients with rheumatoid arthritis. Mod. Rheumatol 17: 531-533. 
Citation: Richi P, Munoz-Fernandez S (2016) What can we do to Prevent Infections in Adult Patients with Rheumatic Diseases?. J Vasc 2: 113. doi:10.4172/2471-9544.100113

Page 5 of 5

28. Fomin I, Caspi D, Levy V, Varsano N, Shalev Y, et al. (2006) Vaccination against influenza in rheumatoid arthritis: the effect of disease modifying drugs, including TNFa blockers. Ann Rheum Dis 65: 191-194.

29. Kapetanovic M, Lars-Erik K, Saxne T, Aktas T, Mörner A, et al. (2014) Impact of anti-rheumatic treatment on immunogenicity of pandemic H1N1 influenza vaccine in patients with arthritis. Arthritis Research \& Therapy 16: R2.

30. Gelinck L, Van der Bijl B, Beyer W, Visser L, Huizinga T, et al. (2008) The effect of anti-tumour necrosis factor a treatment on the antibody response to influenza vaccination. Ann Rheum Dis 67:713-716.

31. Kapetanovic MC, Roseman C, Jönsson G, Truedsson L, Saxne T, et al. (2011) Antibody response is reduced following vaccination with 7-valent conjugate pneumococcal vaccine in adult methotrexate-treated patients with established arthritis, but not those treated with tumor necrosis factor inhibitors. Arthritis Rheum. 63: 3723-3732.

32. Kapetanovic M, Saxne T, Jönsson G, Truedsson L, Geborek P, et al. (2013) Rituximab and abatacept but not tocilizumab impair antibody response to pneumococcal conjugate vaccine in patients with rheumatoid arthritis. Arthritis Research \& Therapy, 15:R171.

33. Salemi S, D’Amelio R (2010) Are anti-infectious vaccinations safe and effective in patients with autoimmunity? Int Rev Immunol 29:270-314.

34. Vaccines That Might Be Indicated For Adults Aged 19 Years or Older Based On Medical and Other Indications (2016) USA. Consulted on 2016-07-01. 